\title{
Complete High Temperature Expansions for One-Loop Finite Temperature Effects
}

\author{
Peter N. Meisinger and Michael C. Ogilvie \\ Dept. of Physics Washington University St. Louis, MO 63130
}

(November 2, 2018)

\begin{abstract}
We develop exact, simple closed form expressions for partition functions associated with relativistic bosons and fermions in odd spatial dimensions. These expressions, valid at high temperature, include the effects of a non-trivial Polyakov loop and generalize well-known high temperature expansions. The key technical point is the proof of a set of Bessel function identities which resum low temperature expansions into high temperature expansions. The complete expressions for these partition functions can be used to obtain one-loop finite temperature contributions to effective potentials, and thus free energies and pressures.
\end{abstract}

\section{INTRODUCTION}

The applications of finite temperature field theory are numerous and diverse [1] 3] For many applications, a hightemperature expansion of one-loop contributions to thermodynamic functions is necessary. A typical one-loop contribution to a $(d+1)$-dimensional effective potential from a bosonic degree of freedom and its antiparticle has the form

$$
V=\frac{2}{\beta} \int \frac{d^{d} k}{(2 \pi)^{d}} \ln \left[1-e^{-\beta \omega_{k}}\right]
$$

where the relativistic energy $\omega_{k}$ is given by $\sqrt{k^{2}+M^{2}}$. In many cases, the mass $M$ is a function of other quantities, most notably vacuum expectation values of fields. When the total effective potential attains its minimum, $V$ may be identified as a contribution to the total free energy, and $-V$ as a contribution to the pressure. A more general case is obtained when there is a non-trivial, but spatially uniform, Polyakov loop as well as a non-zero chemical potential $\mu$.In this case we have

$$
V_{B}(\theta-i \beta \mu)=\frac{1}{\beta} \int \frac{d^{d} k}{(2 \pi)^{d}} \ln \left[1-e^{-\beta \omega_{k}+i \theta+\beta \mu}\right]+\frac{1}{\beta} \int \frac{d^{d} k}{(2 \pi)^{d}} \ln \left[1-e^{-\beta \omega_{k}-i \theta-\beta \mu}\right] .
$$

Note that the effect of a non-trivial Polyakov loop is to add a phase factor $\exp ( \pm i \theta)$ to $\exp (-\beta \omega)$. We will evaluate $V_{B}$ for arbitrary $\theta$. In principle, the effect of $\mu$ can be included by a careful analytic continuation $\theta \rightarrow \theta-i \beta \mu$, but we do not consider it here.

We will develop a high temperature expansion for $V_{B}(\theta)$, valid for $d$ odd, as well as a similar expression for the corresponding fermionic quantity $V_{F}(\theta)$. This derivation is simple and exact, and generalizes the results of Dolan and Jackiw [4], who gave approximate high-temperature expressions for $V_{B}(\theta=0)$ and $V_{F}(\theta=0)$ valid up to order $M^{4} \ln (\beta M)$ in four dimensions. The work of Dolan and Jackiw was extended by Haber and Weldon [5] [6] who gave a complete expression for the Bosonic case $V_{B}$ as an infinite sum over hypergeometric functions. Their work included a non-zero chemical potential. Later work by Actor showed that similar high-temperature expansions could be obtained using zeta-function techniques [7.8. In both cases, higher-order correction terms are given by infinite series in $\beta M$. Our expressions effectively resum these corrections into a simpler form. Analytical results for the case of a non-trivial Polyakov loop, $\theta \neq 0$, were first given in the case $M=0$ by Gross, Pisarski and Yaffe and by Weiss [9]1]. Our work generalizes their results to the case $M \neq 0$. The higher-order terms in our expressions are manifestly periodic in $\theta$. This periodicity is important in the application of these results to our recent work with Miller on models of the deconfinement transition [12]. In this work, the eigenvalues of the Polyakov loop serve as the order parameters for deconfinement, a point of view also emphasized recently by Pisarski [13].

Before beginning the derivation, we give some examples of its application. As a first example, consider a scalar boson in the fundamental representation of an $S U(N)$ gauge group. The Polyakov loop is an $N \times N$ unitary matrix given in general by

$$
P(\vec{x})=\mathcal{T} \exp \left[i \int_{0}^{\beta} d \tau A_{0}(\vec{x}, \tau)\right]
$$


where $\mathcal{T}$ on the right-hand side indicates Euclidean time ordering. Here we assume that the Polyakov loop can be made spatially uniform by an appropriate choice of gauge. A global unitary transformation then puts $P$ into the diagonal form

$$
P_{j k}=\delta_{j k} \exp \left(i \theta_{j}\right)
$$

and the partition function is

$$
\sum_{j} V_{B}\left(\theta_{j}\right)
$$

As a second example, consider the case of the gauge bosons themselves, which lie in the adjoint representation of the gauge group. The Polyakov loop in the adjoint representation is an $\left(N^{2}-1\right) \times\left(N^{2}-1\right)$ matrix. The partition function for the $N^{2}-1$ particles is

$$
s \frac{1}{2} \sum_{j, k=1}^{N}\left(1-\frac{1}{N} \delta_{j k}\right) V_{B}\left(\theta_{j}-\theta_{k}\right)
$$

where the $\delta_{j k}$ removes a singlet contribution, and the factor of $1 / 2$ corrects for overcounting since $V_{B}$ has both a particle and antiparticle contribution. The factor $s$ accounts for spin degeneracy; in $3+1$ dimensions $s=2$, a consequence of the two possible polarization states of gauge bosons .

For our third and final example, consider the evaluation of fermionic partition functions, which can be reduced to the general bosonic problem. A typical fermionic contribution of particle and antiparticle has the form

$$
V_{F}(\theta-i \beta \mu)=-\frac{1}{\beta} \int \frac{d^{d} k}{(2 \pi)^{d}} \ln \left[1+e^{-\beta \omega_{k}+i \theta+\beta \mu}\right]-\frac{1}{\beta} \int \frac{d^{d} k}{(2 \pi)^{d}} \ln \left[1+e^{-\beta \omega_{k}-i \theta-\beta \mu}\right]
$$

which is easily written as

$$
V_{F}(\theta)=-V_{B}(\pi+\theta) .
$$

For fermions in the fundamental representation of $S U(N)$, the partition function is

$$
s \sum_{j} V_{F}\left(\theta_{j}\right)=-s \sum_{j} V_{B}\left(\pi+\theta_{j}\right)
$$

where the factor $s$ again accounts for spin degeneracy.

In section 2 , we review the derivation of low temperature expansions for $V_{B}(\theta)$ and $V_{F}(\theta)$. Section 3 derives the Bessel function identities which convert these low temperature expansions to high temperature expansions. Section 4 applies these identities to the case of three spatial dimensions. A final section gives brief conclusions. There are two appendices.

\section{LOW TEMPERATURE EXPANSION IN $D$ DIMENSIONS}

A low-temperature expansion for $V_{B}(\theta)$ can be generated for arbitrary spatial dimension $d$ by expanding the logarithm and integrating term by term, first over the surface of a $d$-dimensional sphere, and then over a radial degree of freedom $k$ [7], 8 . The result, given in terms of modified Bessel functions [14], is

$$
\begin{aligned}
V_{B}(\theta) & =\frac{1}{\beta} \int \frac{d^{d} k}{(2 \pi)^{d}} \ln \left[1-e^{-\beta \omega_{k}+i \theta}\right]+\frac{1}{\beta} \int \frac{d^{d} k}{(2 \pi)^{d}} \ln \left[1-e^{-\beta \omega_{k}-i \theta}\right] \\
& =-\frac{M^{d / 2+1 / 2}}{2^{d / 2-3 / 2} \pi^{d / 2+1 / 2} \beta^{d / 2+1 / 2}} \sum_{n=1}^{\infty} \frac{1}{n^{d / 2+1 / 2}} K_{(d+1) / 2}(n \beta M) \cos (n \theta)
\end{aligned}
$$

which is derived in detail in Appendix A. Each term in the series represents the contribution of $n$ particles or antiparticles, with a corresponding phase factor of $\exp ( \pm i n \theta)$. If the one-loop finite temperature functional determinant is represented as a functional integral over a space-time variable $x_{\mu}$, the phase factors are associated with paths which wind non-trivially in the Euclidean time direction. 
For fermions, we have

$$
V_{F}(\theta)=\frac{M^{d / 2+1 / 2}}{2^{d / 2-3 / 2} \pi^{d / 2+1 / 2} \beta^{d / 2+1 / 2}} \sum_{n=1}^{\infty} \frac{(-1)^{n}}{n^{d / 2+1 / 2}} K_{(d+1) / 2}(n \beta M) \cos (n \theta) .
$$

In a path integral representation, the factors of $(-1)^{n}$ are a consequence of fermionic antiperiodic boundary conditions. We next derive a set of identities which resum these series for $d$ odd.

\section{BESSEL FUNCTION IDENTITIES}

We will derive a set of identities for sums of the form

$$
\sum_{n=1}^{\infty} \frac{1}{n^{p}} K_{p}(n r) \cos (n \phi)
$$

for $p$ an even integer. Our starting point is the identity

$$
\sum_{p=1}^{\infty} K_{0}(p r) \cos (p \phi)=\frac{1}{2}\left[\gamma+\ln \left(\frac{r}{4 \pi}\right)\right]+\frac{\pi}{2} \sum_{l}^{\prime}\left[\frac{1}{\sqrt{r^{2}+(\phi-2 \pi l)^{2}}}-\frac{1}{2 \pi|l|}\right]
$$

which may be found in [14]; we provide a derivation in Appendix B which provides some physical insight into its origin. The notation $\sum_{l}$ is used to indicate that singular terms, here the $1 /|l|$ term, are omitted when $l=0$.

Using the recursion formula

$$
\frac{d}{d z} K_{\nu}(z)=-K_{\nu-1}(z)-\frac{\nu}{z} K_{\nu}(z)
$$

it follows immediately that

$$
\frac{d}{d z} \sum_{p=1}^{\infty} \frac{z}{p} K_{1}(p z) \cos (p \phi)=-z \sum_{p=1}^{\infty} K_{0}(p z) \cos (p \phi)
$$

This in turn implies that

$$
\sum_{p=1}^{\infty} \frac{1}{p} K_{1}(p z) \cos (p \phi)=-\frac{1}{z} \int d z z\left[\sum_{p=1}^{\infty} K_{0}(p z) \cos (p \phi)\right]+\frac{C(\phi)}{z}
$$

where $C(\phi)$ is an unknown function to be determined. Integration yields immediately

$$
\sum_{p=1}^{\infty} \frac{1}{p} K_{1}(p z) \cos (p \phi)=-\frac{1}{4} z\left[\ln \left(\frac{z}{4 \pi}\right)+\gamma-\frac{1}{2}\right]-\frac{\pi}{2 z} \sum_{l}^{\prime}\left[\sqrt{z^{2}+(\phi-2 \pi l)^{2}}-\frac{z^{2}}{4 \pi|l|}\right]+\frac{C(\phi)}{z} .
$$

The function $C(\phi)$ is determined from the behavior of $K_{\nu}(z)$ for $z \rightarrow 0$

$$
K_{\nu}(z) \rightarrow \frac{1}{2} \Gamma(\nu)\left(\frac{2}{z}\right)^{\nu}
$$

in combination with the standard result 14

$$
\sum_{p=1}^{\infty} \frac{\cos (p \phi)}{p^{2}}=\frac{1}{4} \phi^{2}-\frac{\pi}{2} \phi+\frac{\pi^{2}}{6}
$$

which is valid for $0 \leq \phi<2 \pi$. The right hand side of Eq. (20) is a rescaling of the second Bernoulli polynomial; it can be extended to all real values if $\phi$ is replaced by $\phi \bmod 2 \pi$ on the right hand side of the equation. This implies the leading behavior of the sum as $z \rightarrow 0$ is given by 


$$
\lim _{z \rightarrow 0} z \sum_{p=1}^{\infty} \frac{1}{p} K_{1}(p z) \cos (p \phi)=\sum_{p=1}^{\infty} \frac{\cos (p \phi)}{p^{2}}=\frac{1}{z}\left[\frac{1}{4} \phi^{2}-\frac{\pi}{2} \phi+\frac{\pi^{2}}{6}\right],
$$

giving us finally

$$
\begin{aligned}
\sum_{p=1}^{\infty} \frac{1}{p} K_{1}(p z) \cos (p \phi)= & -\frac{1}{4} z\left[\ln \left(\frac{z}{4 \pi}\right)+\gamma-\frac{1}{2}\right]+\frac{1}{z}\left[\frac{1}{4} \phi_{+}^{2}-\frac{\pi}{2} \phi_{+}+\frac{\pi^{2}}{6}\right] \\
& -\frac{\pi}{2 z} \sum_{l}^{\prime}\left[\sqrt{z^{2}+(\phi-2 \pi l)^{2}}-|\phi-2 \pi l|-\frac{z^{2}}{4 \pi|l|}\right]
\end{aligned}
$$

where we have introduced the notation $\phi_{+}$to represent $\phi \bmod 2 \pi$. When discussing fermions, we will also use $\phi_{-}$to similarly represent an angle chosen to lie between $-\pi$ and $\pi$. Note that the last part of this expression is automatically periodic due to the sum over $l$.

Application of this technique a second time gives

$$
\begin{aligned}
\sum_{p=1}^{\infty} \frac{1}{p^{2}} K_{2}(p z) \cos (p \phi)= & \frac{1}{16} z^{2}\left[\ln \left(\frac{z}{4 \pi}\right)+\gamma-\frac{3}{4}\right]-\frac{1}{2}\left[\frac{1}{4} \phi_{+}^{2}-\frac{\pi}{2} \phi_{+}+\frac{\pi^{2}}{6}\right] \\
& +\frac{2}{z^{2}}\left[\frac{-1}{48} \phi_{+}^{4}+\frac{\pi}{12} \phi_{+}^{3}-\frac{\pi^{2}}{12} \phi_{+}^{2}+\frac{\pi^{4}}{90}\right] \\
& +\frac{\pi}{2 z^{2}} \sum_{l}^{\prime}\left\{\frac{1}{3}\left[z^{2}+(\phi-2 \pi l)^{2}\right]^{3 / 2}-\frac{1}{3}|\phi-2 \pi l|^{3}-\frac{1}{2}|\phi-2 l \pi| z^{2}-\frac{z^{4}}{16 \pi|l|}\right\}
\end{aligned}
$$

which is needed for the case $d=3$. We have used the standard result 14]

$$
\sum_{p=1}^{\infty} \frac{\cos (p \phi)}{p^{4}}=\frac{-1}{48} \phi_{+}^{4}+\frac{\pi}{12} \phi_{+}^{3}-\frac{\pi^{2}}{12} \phi_{+}^{2}+\frac{\pi^{4}}{90} .
$$

Formulas appropriate for $d=5,7, .$. can also be derived in the same manner.

\section{HIGH-TEMPERATURE EXPANSIONS FOR $D=3$}

We now can write complete expressions for $V_{B}(\theta)$ and $V_{F}(\theta)$ in three spatial dimensions:

$$
\begin{aligned}
V_{B}(\theta)= & -\frac{M^{2}}{\pi^{2} \beta^{2}} \sum_{n=1}^{\infty} \frac{1}{n^{2}} K_{2}(n \beta M) \cos (n \theta) \\
= & -\frac{2}{\pi^{2} \beta^{4}}\left[\frac{\pi^{4}}{90}-\frac{1}{48} \theta_{+}^{4}+\frac{\pi}{12} \theta_{+}^{3}-\frac{\pi^{2}}{12} \theta_{+}^{2}\right]+\frac{M^{2}}{2 \pi^{2} \beta^{2}}\left[\frac{1}{4} \theta_{+}^{2}-\frac{\pi}{2} \theta_{+}+\frac{\pi^{2}}{6}\right] \\
& -\frac{1}{2 \pi \beta^{4}} \sum_{l}^{\prime}\left\{\frac{1}{3}\left[(\beta M)^{2}+(\theta-2 \pi l)^{2}\right]^{3 / 2}-\frac{1}{3}|\theta-2 \pi l|^{3}-\frac{1}{2}|\theta-2 l \pi| \beta^{2} M^{2}-\frac{(\beta M)^{4}}{16 \pi|l|}\right\} \\
& -\frac{M^{4}}{16 \pi^{2}}\left[\ln \left(\frac{\beta M}{4 \pi}\right)+\gamma-\frac{3}{4}\right] .
\end{aligned}
$$

Parts of this complete expression have been known for some time. For $\theta=0$, the leading behavior is

$$
V_{B}(\theta=0) \approx-2 \frac{\pi^{2}}{90 \beta^{4}}+\frac{M^{2}}{12 \beta^{2}}-\frac{M^{3}}{6 \pi \beta}-\frac{M^{4}}{16 \pi^{2}}\left[\ln \left(\frac{\beta M}{4 \pi}\right)+\gamma-\frac{3}{4}\right]
$$

an expression first derived by Dolan and Jackiw $\left[\right.$. The leading $T^{4}$ behavior for $\theta \neq 0$ was first derived by Gross, Pisarski and Yaffe and Weiss for $S U(N)$ gauge bosons and for massless fermions [9 11].

Each of the four terms deserves comment. The first is the blackbody free energy for two degrees of freedom, and depends only on the temperature and the angle $\theta$. The second term, which is the leading correction due to the mass 
$M$, often appears in discussions of symmetry restoration at high temperatures with $\theta=0$. For example, suppose we are calculating the effective potential for a complex scalar field $\Phi$. The mass $M^{2}$ is given by the second derivative of the classical potential, $\partial^{2} U / \partial \Phi^{*} \partial \Phi$, and depends on the expectation value of the field $\Phi$. If $U$ has the form $U=-m^{2} \Phi^{*} \Phi+\lambda\left(\Phi^{*} \Phi\right)^{2}$, then $M^{2}=-m^{2}+4 \lambda \Phi^{*} \Phi$. For $m^{2}>0$, the $U(1)$ symmetry is spontaneously broken at low temperature. At high temperature, the $M^{2} / 12 \beta^{2}$ term generates a positive mass for the $\Phi$ field of order $T$, restoring the symmetry. The third term in $V_{B}(\theta)$ is linear in $T$, and non-analytic in $M^{2}$ for $\theta=0$. It is closely associated with the $n=0$ Matsubara mode, which is the most infrared singular contribution to a finite temperature functional determinant. This term is responsible for non-analytic behavior in finite temperature perturbation theory via the summation of ring diagrams. For example, in a scalar theory it gives rise to the $\lambda^{3 / 2}$ contribution to the free energy; in QED, the contribution is $e^{3}$ [1] Note how subtractions occur in the $l \neq 0$ parts of this term to keep these parts subleading. The last term is logarithmic in the dimensionless combination $\beta M$ and independent of $\theta$. In calculations of effective potentials, it typically combines with zero-temperature logarithms in such a way that the temperature $T$ sets the scale of running coupling constants at high $T$.

From the basic result for $V_{B}$, we can build other results. Consider a complex scalar field in the fundamental representation of $S U(N)$. The partition function in a constant background Polyakov loop is given by

$$
\begin{aligned}
V_{F T}= & \sum_{j} V_{B}\left(\theta_{j}\right) \\
= & -\frac{\pi^{2} N}{45 \beta^{4}}+\frac{2}{\pi^{2} \beta^{4}} \sum_{j}\left[\frac{1}{48} \theta_{j}^{4}-\frac{\pi}{12} \theta_{j}^{3}+\frac{\pi^{2}}{12} \theta_{j}^{2}\right] \\
& +\frac{N M^{2}}{12 \beta^{2}}+\frac{M^{2}}{2 \pi^{2} \beta^{2}} \sum_{j}\left[\frac{1}{4} \theta_{j}^{2}-\frac{\pi}{2} \theta_{j}\right] \\
& -\frac{1}{2 \pi \beta^{4}} \sum_{j, l}^{\prime}\left\{\frac{1}{3}\left[(\beta M)^{2}+\left(\theta_{j}-2 \pi l\right)^{2}\right]^{3 / 2}-\frac{1}{3}\left|\theta_{j}-2 \pi l\right|^{3}-\frac{1}{2}\left|\theta_{j}-2 l \pi\right| \beta^{2} M^{2}-\frac{(\beta M)^{4}}{16 \pi|l|}\right\} \\
& -\frac{N M^{4}}{16 \pi^{2}}\left[\ln \left(\frac{\beta M}{4 \pi}\right)+\gamma-\frac{3}{4}\right]
\end{aligned}
$$

where we assume all $\theta$ 's are chosen to lie between 0 and $2 \pi$ in accordance with the convention for $\theta_{+}$. A similar expression holds for bosons in the adjoint representation.

For fermions, we have similarly

$$
\begin{aligned}
V_{F}(\theta)= & \frac{2}{\pi^{2} \beta^{4}}\left[-\frac{7}{720} \pi^{4}+\frac{1}{24} \pi^{2} \theta_{-}^{2}-\frac{1}{48} \theta_{-}^{4}\right]+\frac{M^{2}}{2 \pi^{2} \beta^{2}}\left[\frac{1}{12} \pi^{2}-\frac{1}{4} \theta_{-}^{2}\right] \\
& +\frac{1}{2 \pi \beta^{4}} \sum_{l}^{\prime}\left\{\frac{1}{3}\left[(\beta M)^{2}+(\theta-(2 l-1) \pi)^{2}\right]^{3 / 2}-\frac{1}{3}|\theta-(2 l-1) \pi|^{3}-\frac{1}{2}|\theta-(2 l-1) \pi| \beta^{2} M^{2}-\frac{(\beta M)^{4}}{16 \pi|l|}\right\} \\
& +\frac{M^{4}}{16 \pi^{2}}\left[\ln \left(\frac{\beta M}{4 \pi}\right)+\gamma-\frac{3}{4}\right]
\end{aligned}
$$

with $\theta_{-}$now used. For fermions in the fundamental representation of $S U(N)$, we may write

$$
\begin{aligned}
V_{F T}= & -\frac{7 \pi^{2} N}{180 \beta^{4}}+\sum_{j} \frac{1}{12 \pi^{2} \beta^{4}}\left[2 \pi^{2} \theta_{j}^{2}-\theta_{j}^{4}\right]+\frac{N M^{2}}{12 \beta^{2}}-\sum_{j} \frac{M^{2}}{4 \pi^{2} \beta^{2}} \theta_{j}^{2} \\
& +\frac{1}{\pi \beta^{4}} \sum_{j, l}^{\prime}\left\{\frac{1}{3}\left[(\beta M)^{2}+\left(\theta_{j}-(2 l-1) \pi\right)^{2}\right]^{3 / 2}-\frac{1}{3}\left|\theta_{j}-(2 l-1) \pi\right|^{3}-\frac{1}{2}\left|\theta_{j}-(2 l-1) \pi\right| \beta^{2} M^{2}-\frac{(\beta M)^{4}}{16 \pi|l|}\right\} \\
& +\frac{N M^{4}}{8 \pi^{2}}\left[\ln \left(\frac{\beta M}{4 \pi}\right)+\gamma-\frac{3}{4}\right] .
\end{aligned}
$$

where the angles $\theta_{j}$ must now be chosen to lie between $-\pi$ and $\pi$. 


\section{CONCLUSIONS}

We have found complete, simple expressions for $V_{B}(\theta)$ and $V_{F}(\theta)$ in the high-temperature limit which generalize previously known expressions. Not only are the expressions simple, their derivation is direct and relatively elementary. Our formulae reflect in a direct way periodicity in $\theta$, a property which is lost when analytically continuing power series in $\beta \mu$ to $\beta \mu+i \theta$.

As a practical matter, it is natural to ask how accurate both the low- and high-temperature expansions are. The low temperature expansion for $V_{B}(\theta)$ is an infinite series in $n$; using the first 10 terms in the series gives an accuracy better than 1 part in $10^{3}$ over the entire range 0 to $2 \pi$ for temperatures $T \leq 0.25 \mathrm{M}$. The high temperature expansion also involves an infinite series, in the the parameter $l$. In comparison, the high temperature expansion is within $5 \%$ of the exact answer for all values of $\theta$ at $T=0.5 \mathrm{M}$ when terms up to $|l|=10$ are included. The accuracy improves substantially as $T$ increases. Both expansions are more accurate when restricted to $\theta=0$.

Our primary interest in these results lies in their application to the study of systems where a non-trivial Polyakov loop is expected to occur. The foremost physical example is QCD at finite temperature. The high-temperature form of $V_{B}(\theta)$ suggests that the Bernoulli polynomials appear naturally in the free energy of $S U(N)$ gauge theories with a non-trivial Polyakov loop, essentially as polynomials in the Polyakov loop eigenvalues. In our recent work with Miller [12], we have used this observation to construct a phenomenological free energy for the quark-gluon plasma which reproduces much of the thermodynamic observed in lattice simulations. One can also apply the results obtained here to the Savvidy model at finite temperature [15, 16]. Savvidy originally proposed a model of the QCD vacuum in which gluons moved in a constant chromomagnetic field [17]. Using low-temperature expansions, we have shown that a confining state, where the Polyakov loop expectation value is zero, minimizes the free energy. Using the Bessel function identities proven here, we have recently [18] developed a high-temperature expansion for this model which shows that a non-zero Polyakov line is favored at high temperature.

\section{APPENDIX A: DERIVATION OF LOW TEMPERATURE EXPANSIONS}

We begin by expanding the logarithms and performing the angular integrations:

$$
\begin{aligned}
V_{B}(\theta) & =\frac{1}{\beta} \int \frac{d^{d} k}{(2 \pi)^{d}} \ln \left[1-e^{-\beta \omega_{k}+i \theta}\right]+\frac{1}{\beta} \int \frac{d^{d} k}{(2 \pi)^{d}} \ln \left[1-e^{-\beta \omega_{k}-i \theta}\right] \\
& =-\frac{4 \pi^{d / 2}}{\Gamma(d / 2)(2 \pi)^{d} \beta} \int d k k^{d-1} \sum_{n=1}^{\infty} \frac{1}{n} e^{-n \beta \omega_{k}} \cos (n \theta) .
\end{aligned}
$$

The standard substitution $k=M \sinh (t)$ gives

$$
\begin{aligned}
V_{B}(\theta) & =-\frac{4 \pi^{d / 2}}{\Gamma(d / 2)(2 \pi)^{d} \beta} \sum_{n=1}^{\infty} \frac{\cos (n \theta)}{n} M^{d} \int_{0}^{\infty} d t \cosh t \sinh t^{d-1} e^{-n \beta M \cosh t} \\
& =\frac{4 \pi^{d / 2}}{\Gamma(d / 2)(2 \pi)^{d}} \sum_{n=1}^{\infty} \frac{\cos (n \theta)}{n^{2} \beta^{2}} M^{d} \frac{d}{d M} \int_{0}^{\infty} d t \sinh t^{d-1} e^{-n \beta M \cosh t} \\
& =\frac{4 \pi^{d / 2}}{\Gamma(d / 2)(2 \pi)^{d}} \sum_{n=1}^{\infty} \frac{\cos (n \theta)}{n^{2} \beta^{2}} M^{d} \frac{d}{d M}\left[\frac{\Gamma(d / 2)}{\sqrt{\pi}}\left(\frac{2}{n \beta M}\right)^{(d-1) / 2} K_{(d-1) / 2}(n \beta M)\right]
\end{aligned}
$$

This can in turn be reduced using standard recursion relations for modified Bessel functions:

$$
\begin{aligned}
V_{B}(\theta) & =\frac{4 \pi^{(d-1) / 2}}{(2 \pi)^{d} \beta} M^{d} \sum_{n=1}^{\infty} \frac{\cos (n \theta)}{n} \frac{d}{d z}\left[\left(\frac{2}{z}\right)^{\nu} K_{\nu}(z)\right]_{z=n \beta M, \nu=(d-1) / 2} \\
& =\frac{4 \pi^{(d-1) / 2}}{(2 \pi)^{d} \beta} M^{d} \sum_{n=1}^{\infty} \frac{\cos (n \theta)}{n}\left[\left(\frac{2}{z}\right)^{\nu}\left(\frac{d K_{\nu}(z)}{d z}-\frac{\nu}{z} K_{\nu}(z)\right)\right]_{z=n \beta M, \nu=(d-1) / 2} \\
& =\frac{4 \pi^{(d-1) / 2}}{(2 \pi)^{d} \beta} M^{d} \sum_{n=1}^{\infty} \frac{\cos (n \theta)}{n}\left[-\left(\frac{2}{z}\right)^{\nu} K_{\nu+1}(z)\right]_{z=n \beta M, \nu=(d-1) / 2}
\end{aligned}
$$




$$
\begin{aligned}
& =-\frac{4 \pi^{(d-1) / 2}}{(2 \pi)^{d} \beta} M^{d} \sum_{n=1}^{\infty} \frac{\cos (n \theta)}{n}\left(\frac{2}{n \beta M}\right)^{(d-1) / 2} K_{(d+1) / 2}(n \beta M) \\
& =-\frac{M^{d / 2+1 / 2}}{2^{d / 2-3 / 2} \pi^{d / 2+1 / 2} \beta^{d / 2+1 / 2}} \sum_{n=1}^{\infty} \frac{\cos (n \theta)}{n^{d / 2+1 / 2}} K_{(d+1) / 2}(n \beta M)
\end{aligned}
$$

\section{APPENDIX B: PROOF OF A BESSEL FUNCTION IDENTITY}

In this appendix, we prove the Bessel function identity

$$
\sum_{m=1}^{\infty} K_{0}(m r) \cos (m \phi)=\frac{1}{2}\left[\ln \left(\frac{r}{4 \pi}\right)+\gamma\right]+\frac{\pi}{2} \sum_{l}^{\prime}\left[\frac{1}{\sqrt{r^{2}+(\phi-2 \pi l)^{2}}}-\frac{1}{2 \pi|l|}\right] .
$$

Using a standard integral representation [14]

$$
K_{0}(p z)=\int_{0}^{\infty} d t \frac{\cos (p t)}{\sqrt{t^{2}+z^{2}}}
$$

we have

$$
\begin{aligned}
& \sum_{m=1}^{\infty} \cos (m \phi) K_{0}(m r) \\
= & \sum_{m=1}^{\infty} \cos (m \phi) \int_{0}^{\infty} d k_{x} \frac{\cos \left(k_{x} r\right)}{\sqrt{k_{x}^{2}+m^{2}}} \\
= & \frac{1}{2} \sum_{m=1}^{\infty} \cos (m \phi) \int d k_{x} \frac{1}{\sqrt{k_{x}^{2}+m^{2}}} e^{i k_{x} r} \\
= & \frac{1}{4 \pi} \sum_{m \neq 0} \int d k_{x} d k_{y} \frac{1}{k_{x}^{2}+k_{y}^{2}+m^{2}} e^{i k_{x} r+i m \phi}
\end{aligned}
$$

We introduce a regulating mass $\mu$, which will be taken to zero at the end of the calculation, obtaining

$$
\frac{1}{4 \pi} \sum_{m \neq 0} \int d k_{x} d k_{y} \frac{1}{k_{x}^{2}+k_{y}^{2}+m^{2}+\mu^{2}} e^{i k_{x} r+i m \phi}
$$

We add and subtract the divergent $m=0$ term

$$
\begin{aligned}
& \frac{1}{4 \pi} \sum_{m} \int d k_{x} d k_{y} \frac{1}{k_{x}^{2}+k_{y}^{2}+m^{2}+\mu^{2}} e^{i k_{x} r+i m \phi}-\frac{1}{4 \pi} \int d k_{x} d k_{y} \frac{1}{k_{x}^{2}+k_{y}^{2}+\mu^{2}} e^{i k_{x} r} \\
= & \frac{1}{4 \pi} \sum_{m} \int d k_{x} d k_{y} d k_{z} \frac{1}{k_{x}^{2}+k_{y}^{2}+k_{z}^{2}+\mu^{2}} \delta\left(k_{z}-m\right) e^{i k_{x} r+i k_{z} \phi}-\frac{1}{4 \pi} \int d k_{x} d k_{y} \frac{1}{k_{x}^{2}+k_{y}^{2}+\mu^{2}} e^{i k_{x} r}
\end{aligned}
$$

Using the Poisson summation technique in the form $\sum_{m} \delta\left(k_{z}-m\right)=\sum_{n} \exp \left(-2 \pi i n k_{z}\right)$, we obtain

$$
\frac{1}{4 \pi} \sum_{n} \int d k_{x} d k_{y} d k_{z} \frac{1}{k_{x}^{2}+k_{y}^{2}+k_{z}^{2}+\mu^{2}} e^{i k_{x} r+i k_{z}(\phi-2 \pi n)}-\frac{1}{4 \pi} \int d k_{x} d k_{y} \frac{1}{k_{x}^{2}+k_{y}^{2}+\mu^{2}} e^{i k_{x} r}
$$

which reads in a compact notation

$$
\frac{\pi}{2} \sum_{n} \int \frac{d^{3} k}{(2 \pi)^{3}} \frac{4 \pi}{k^{2}+\mu^{2}} e^{i k_{x} r+i k_{z}(\phi-2 \pi n)}-\frac{1}{4 \pi} \int d^{2} k \frac{1}{k^{2}+\mu^{2}} e^{i k_{x} r}
$$

The first integral gives a sum of screened Coulomb, or Yukawa, potentials 


$$
\frac{\pi}{2} \sum_{n}\left[\frac{e^{-\mu \sqrt{r^{2}+(\phi-2 \pi n)^{2}}}}{\sqrt{r^{2}+(\phi-2 \pi n)^{2}}}\right]-\frac{1}{4 \pi} \int d^{2} k \frac{1}{k^{2}+\mu^{2}} e^{i k_{x} r}
$$

and both terms appear to be problematic as $\mu \rightarrow 0$. The first term can be made finite in this limit by subtracting the contribution at $r=\phi=0$ for $n \neq 0$. Using the notation $\sum_{n}{ }^{\prime}$ to denote a summation over all $n$ with the omission of the singular term when $n=0$, we have

$$
\frac{\pi}{2} \sum_{n} \cdot\left[\frac{e^{-\mu \sqrt{r^{2}+(\phi-2 \pi n)^{2}}}}{\sqrt{r^{2}+(\phi-2 \pi n)^{2}}}-\frac{e^{-\mu 2 \pi|n|}}{2 \pi|n|}\right]+\left[\frac{\pi}{2} \sum_{n}{ }^{\prime} \frac{e^{-\mu 2 \pi|n|}}{2 \pi|n|}-\frac{1}{4 \pi} \int d^{2} k \frac{1}{k^{2}+\mu^{2}} e^{i k_{x} r}\right] .
$$

The second term in brackets can be evaluated by summing the series

$$
\frac{\pi}{2} \sum_{n}^{\prime} \frac{e^{-\mu 2 \pi|n|}}{2 \pi|n|}=-\frac{1}{2} \ln \left[1-e^{-2 \pi \mu}\right]
$$

and using the identity

$$
-\frac{1}{4 \pi} \int d^{2} k \frac{1}{k^{2}+\mu^{2}} e^{i k_{x} r}=-\frac{1}{2} K_{0}(\mu r)
$$

so we have

$$
\frac{\pi}{2} \sum_{n} \cdot\left[\frac{e^{-\mu \sqrt{r^{2}+(\phi-2 \pi n)^{2}}}}{\sqrt{r^{2}+(\phi-2 \pi n)^{2}}}-\frac{e^{-\mu 2 \pi|n|}}{2 \pi|n|}\right]+\left[-\frac{1}{2} \ln \left[1-e^{-2 \pi \mu}\right]-\frac{1}{2} K_{0}(\mu r)\right] .
$$

In the limit $\mu \rightarrow 0$, the second term in brackets gives

$$
\begin{aligned}
& -\frac{1}{2} \ln \left[1-e^{-2 \pi \mu}\right]-\frac{1}{2} K_{0}(\mu r) \\
\rightarrow & -\frac{1}{2} \ln [2 \pi \mu]+\frac{1}{2} \ln \left(\frac{\mu r}{2}\right)-\frac{1}{2} \psi(1)=\frac{1}{2} \ln \left(\frac{r}{4 \pi}\right)+\frac{1}{2} \gamma
\end{aligned}
$$

so we finally obtain

$$
\sum_{m=1}^{\infty} K_{0}(m r) \cos (m \phi)=\frac{\pi}{2} \sum_{n}^{\prime}\left[\frac{1}{\sqrt{r^{2}+(\phi-2 \pi n)^{2}}}-\frac{1}{2 \pi|n|}\right]+\frac{1}{2} \ln \left(\frac{r}{4 \pi}\right)+\frac{1}{2} \gamma .
$$

[1] J. I. Kapusta, Finite Temperature Field Theory (Cambridge , 1989).

[2] M. Le Bellac, Thermal Field Theory (Cambridge , 1996).

[3] A. Das, Finite Temperature Field Theory (World Scientific, Singapore, 1997)

[4] L. Dolan and R. Jackiw, Phys. Rev. D 9, 3320 (1974).

[5] H. E. Haber and H. A. Weldon, Phys. Rev. D 25, 502 (1982).

[6] H. E. Haber and H. A. Weldon, J. Math. Phys. 23, 1852 (1982).

[7] A. Actor, Nucl. Phys. B 265, 689 (1986).

[8] A. Actor, Fortsch. Phys. 35, 793 (1987).

[9] D. J. Gross, R. D. Pisarski and L. G. Yaffe, Rev. Mod. Phys. 53, 43 (1981).

[10] N. Weiss, Phys. Rev. D 24, 475 (1981).

[11] N. Weiss, Phys. Rev. D 25, 2667 (1982).

[12] P. N. Meisinger, T. R. Miller and M. C. Ogilvie, arXiv:hep-ph/0108009.

[13] R. D. Pisarski, Phys. Rev. D 62, 111501 (2000) hep-ph/0006205]. 
[14] I. Gradshteyn and I. Ryzhik, Table of Integrals, Series, and Products, 5th ed. (Academic, San Diego, 1994).

[15] A. O. Starinets, A. S. Vshivtsev and V. C. Zhukovsky, Phys. Lett. B 322, 403 (1994).

[16] P. N. Meisinger and M. C. Ogilvie, Phys. Lett. B 407, 297 (1997) arXiv:hep-lat/9703009.

[17] G. K. Savvidy, Phys. Lett. B 71, 133 (1977).

[18] P. N. Meisinger and M. C. Ogilvie, in preparation. 